\title{
Narrow Convergence in Spaces of Set-Valued Measures by
}

\author{
Kenny Koffi SIGGINI \\ Presented by Czesław RYLL-NARDZEWSKI
}

Summary. We prove an analogue of Topsøe's criterion for relative compactness of a family of probability measures which are regular with respect to a family sets. We consider measures whose values are compact convex sets in a locally convex linear topological space.

Introduction. Let $T$ be an abstract set, $\mathcal{K}$ a family of subsets of $T$, and $(E, F)$ a dual pair of real vector spaces, with $E$ endowed with the weak topology $\sigma(E, F)$. Let $\mathrm{cc}(E, F)$ be the set of all convex compact non-empty subsets of $E$, and $\widetilde{M}_{+}(T, \mathcal{K}, \operatorname{cc}(E, F))$ the set of $\mathcal{K}$-inner regular positive set-valued measures defined on a $\sigma$-field $\mathcal{B}$ of subsets of $T$ and with values in $\operatorname{cc}(E, F)$. We denote by $M_{+}(T, \mathcal{K})$ the set of $\mathcal{K}$-inner regular non-negative measures defined on $\mathcal{B}$ provided with the topology of weak convergence. Prokhorov [11] has proved that if $T$ is a Polish space and $\mathcal{B}$ the set of Borel subsets, then the relatively compact subsets of $M_{+}(T, \mathcal{K})$ are precisely the tight ones. But this result is not valid for all topological space (see e.g. [5], [10], [18]). In [16] Topsøe has characterized the relatively compact subsets of $M_{+}(T, \mathcal{K})$ in general situations. Before and after Topsøe's paper there were others (e.g. [1], [3], [18], [5]-[10]). In this paper we generalize to the space $\widetilde{M}_{+}(T, \mathcal{K}, \operatorname{cc}(E, F))$ the criterion of Topsøe (Theorem 2.1). In addition, we prove a result (Theorem 3.3) analogous to Theorem 8.1 in [17, p. 40].

\section{Preliminaries}

1.1. We denote by $T$ an abstract set; $\mathcal{G}$ and $\mathcal{K}$ are families of subsets of $T$. We let $\mathcal{B}$ denote the smallest $\sigma$-field containing every set $A \subseteq T$ for

2000 Mathematics Subject Classification: Primary 28B20.

Key words and phrases: weak set-valued measure, set-valued measure, narrow convergence. 
which $K \cap A \in \mathcal{K}$ for all $K \in \mathcal{K}$. The family $\mathcal{K}$ is said to be semicompact if every countable subfamily of $\mathcal{K}$ with the finite intersection property has a non-empty intersection. We shall say that $\mathcal{G}$ separates the sets in $\mathcal{K}$ if for any pair $K, K^{\prime}$ of disjoint sets in $\mathcal{K}$ we can find a pair $G, G^{\prime}$ of disjoint sets in $\mathcal{G}$ such that $K \subset G$ and $K^{\prime} \subset G^{\prime}$.

Let $\mathcal{G}^{\prime}$ be a family of subsets of $T$ such that $\mathcal{G}^{\prime} \subseteq \mathcal{G}$. We shall say that $\mathcal{G}^{\prime}$ dominates $\mathcal{K}$ and write $\mathcal{G}^{\prime} \succ \mathcal{K}$ if for any $K \in \mathcal{K}$ there exists $G^{\prime} \in \mathcal{G}^{\prime}$ such that $K \subseteq G^{\prime}$.

1.2. Nets on $T$. Let $X$ be a non-empty subset of $T$ and $\left(x_{i}\right)_{i \in I}$ be a net on $T$. We say that $x_{i} \in X$ eventually if there exists $i \in I$ such that $x_{j} \in X$ for every $j \in I$ with $j \geq i$. A net $\left(x_{i}\right)_{i \in I}$ on $T$ is universal if, for every subset $X \subset T$ either $x_{i} \in X$ eventually or $x_{i} \in T \backslash X$ eventually.

1.3. The space $\operatorname{cc}(E, F)$. Let $(E, F)$ be a dual pair of real vector spaces, with $E$ and $F$ endowed with the weak topologies $\sigma(E, F)$ and $\sigma(F, E)$ respectively. If $X$ and $Y$ are subsets of $E$, we denote by $X+Y$ the subset of $E$ consisting of all elements of the form $x+y$, where $x \in X$ and $y \in Y$. The closed convex hull of $X$ is denoted by $\overline{c o} X$, the polar of $X$ by $\stackrel{\circ}{X}$, and the closure of $X$ by $\operatorname{cl} X$. The support function of $X$ is the map

$$
\delta^{*}(\cdot \mid X): F \rightarrow[-\infty,+\infty], \quad y \mapsto \delta^{*}(y \mid X)=\sup \{y(x) ; x \in X\} .
$$

We denote by $\operatorname{cc}(E, F)$ the set of all $\sigma(E, F)$-compact non-empty convex subsets of $E$. We equip cc $(E, F)$ with the Hausdorff topology. Let $C \in \operatorname{cc}(E, F)$, $\beta(o)$ a base of neighbourhoods of $o$ in $E, V \in \beta(o)$, and $\varepsilon>0$. The set

$$
W_{(V, \varepsilon, C)}=\left\{C^{\prime} \in \operatorname{cc}(E, F) ; \sup _{y \in V}\left|\delta^{*}(y \mid C)-\delta^{*}\left(y \mid C^{\prime}\right)\right|<\varepsilon\right\}
$$

is a neighbourhood of $C$. The family $\left\{W_{(V, \varepsilon, C)} ; V \in \beta(o)\right.$ and $\left.\varepsilon>0\right\}$ is a base of neighborhoods of $C$. The space $\operatorname{cc}(E, F)$ is a completely regular topological space ([2, Theorem II.19]).

1.4. Set-valued measures. Let $M$ be a map from $\mathcal{B}$ to $\operatorname{cc}(E, F)$. The map $M$ is called additive if $M(A \cup B)=M(A)+M(B)$ for any disjoint sets $A, B$ in $\mathcal{B}$; monotone if $M(\emptyset)=\{o\}$ and $M(A) \subseteq M(B)$ for all $A$ and $B$ in $\mathcal{B}$ such that $A \subseteq B$; and positive if $M(\emptyset)=\{o\}$ and $o \in M(A)$ for all $A \in \mathcal{B}$. We say that $M$ is a weak set-valued measure if $M$ is additive and for every $y \in F$ the map $A \mapsto \delta^{*}(y \mid M(A))$ from $\mathcal{B}$ to $\mathbb{R}$ is a $\sigma$-additive measure. A positive weak set-valued measure is $\mathcal{K}$-inner regular if for every $A \in \mathcal{B}, M(A)=\overline{\mathrm{co}} \bigcup\{M(K) ; K \subset A, K \in \mathcal{K}\}$. Note that a positive additive map $M: \mathcal{B} \rightarrow \operatorname{cc}(E, F)$ is monotone. Indeed, if $A, B \in \mathcal{B}$ and $A \subseteq B$, then $M(B)=M(A)+M(B \backslash A)$. Hence $M(A)=M(A)+\{o\} \subset M(B)$ since $o \in M(B \backslash A)$. 
1.5. Set-valued integral. An integration theory for positive weak setvalued measures is developed in [15]. Let us only recall the following definitions and results. Assume that $M: \mathcal{B} \rightarrow \operatorname{cc}(E, F)$ is a positive weak set-valued measure. If $h$ is a positive simple function defined on $T$ (i.e. $h=\sum_{i=1}^{n} \alpha_{i} 1_{A_{i}}$ where $\alpha_{i} \geq 0, A_{i} \in \mathcal{B}$ and $\left\{A_{1}, \ldots, A_{n}\right\}$ is a partition of $T$ ) then the integral of $h$ with respect to $M$ is defined by $\int h M=$ $\sum_{i=1}^{n} \alpha_{i} M\left(A_{i}\right)$. If $f$ is a positive measurable function with respect to $\mathcal{B}$ and the Borel field of $\mathbb{R}$, there exists an increasing sequence $\left(h_{n}\right)$ of simple functions such that $f=\sup \left\{h_{n} ; n \in \mathbb{N}\right\}$. The integral of $f$ is defined by $\int f M=\overline{\mathrm{co}} \bigcup\left\{\int h_{n} M ; n \in \mathbb{N}\right\}$. We have $\delta^{*}\left(y \mid \int f M\right)=\int f \delta^{*}(y \mid M(\cdot))$ for every $y \in F$. If $f$ is bounded we have $\int f M \in \operatorname{cc}(E, F)$. If $f$ and $g$ are measurable functions and $f \leq g$ then $\int f M \subseteq \int g M$.

1.6. Topologies on $\widetilde{M}_{+}(T, \operatorname{cc}(E, F))$. We denote by $\widetilde{M}_{+}(T, \operatorname{cc}(E, F))$ the set of all positive weak set-valued measures from $\mathcal{B}$ to $\operatorname{cc}(E, F)$ and by $\widetilde{M}_{+}(T, \mathcal{K}, \operatorname{cc}(E, F))$ the subset of $\widetilde{M}_{+}(T, \operatorname{cc}(E, F))$ consisting of all $\mathcal{K}$-inner regular elements. In $\widetilde{M}_{+}(T, \operatorname{cc}(E, F))$ we define the following topologies.

The weak narrow topology (wn-topology) on $\widetilde{M}_{+}(T, \operatorname{cc}(E, F))$ is the weakest topology for which the map $M \mapsto M(T)$ is continuous and all maps $M \mapsto \delta^{*}(y \mid M(G))$ are lower semicontinuous for every $G \in \mathcal{G}$ and $y \in F$.

The strong narrow topology (sn-topology) on $\widetilde{M}_{+}(T, \operatorname{cc}(E, F))$ is the weakest topology for which the map $M \mapsto M(T)$ is continuous and all maps $M \mapsto M(G)$ are lower semicontinuous for every $G \in \mathcal{G}$.

Let $M \in \widetilde{M}_{+}(T, \operatorname{cc}(E, F))$ and let $\left(M_{i}\right)_{i \in I}$ be a net on $\left.\widetilde{M}_{+}(T, \operatorname{cc}(E, F))\right)$. Then $\left(M_{i}\right)$ converges to $M$ in the wn-topology if and only if $\left(M_{i}(T)\right)$ converges to $M(T)$ in $\operatorname{cc}(E, F)$ and $\liminf _{i} \delta^{*}\left(y \mid M_{i}(G)\right) \geq \delta^{*}(y \mid M(G))$ for all $y \in F$ and $G \in \mathcal{G}$; and $\left(M_{i}\right)$ converges to $M$ in the sn-topology if and only if $\left(M_{i}(T)\right)$ converges to $M(T)$ in $\operatorname{cc}(E, F)$ and for every $G \in \mathcal{G}$ and every open subset $O$ of $E$ such that $M(G) \cap O \neq \emptyset$ there exists $i_{0} \in I$ such that $M_{i}(G) \cap O \neq \emptyset$ for every $i \in I$ with $i \geq i_{0}$. The subset $\widetilde{M}_{+}(T, \mathcal{K}, \operatorname{cc}(E, F))$ will be considered as a subspace of $\widetilde{M}_{+}(T, \operatorname{cc}(E, F))$.

Consider now the following axioms on $\mathcal{K}$ and $\mathcal{G}$, introduced by Topsøe [16].

(i) $\mathcal{K}$ is closed under finite unions and countable intersections, and $\emptyset \in \mathcal{K}$.

(ii) $\mathcal{G}$ is closed under finite unions and finite intersections, and $\emptyset \in \mathcal{G}$.

(iii) For every $K \in \mathcal{K}$ and every $G \in \mathcal{G}, K \backslash G \in \mathcal{K}$.

(iv) $\mathcal{G}$ separates the sets in $\mathcal{K}$.

(v) $\mathcal{K}$ is semicompact.

Note that (i) and (iv) imply that $\mathcal{G}$ dominates $\mathcal{K}$. 
1.7. Topological case. Assume now that $T$ is a Hausdorff topological space. We then denote by $\mathcal{K}(T), \mathcal{G}(T)$ and $\mathcal{B}(T)$ the families of compact subsets, open subsets, and Borel subsets of $T$, respectively. Now $\widetilde{M}_{+}(T, \operatorname{cc}(E, F))$ denotes the set of positive weak set-valued measures defined on $\mathcal{B}(T)$. The $w n$-topology and $s n$-topology are defined by means of $\mathcal{G}(T)$. Generally $\mathcal{K}(T)$, $\mathcal{G}(T), \mathcal{B}(T)$ replace $\mathcal{K}, \mathcal{G}$ and $\mathcal{B}$ respectively. We denote by $C_{+}(T)$ the set of non-negative bounded continuous functions defined on $T$. In view of [17, Theorem 8.1 p. 40] if $T$ is a completely regular space then a net $\left(M_{i}\right)$ on $\widetilde{M}_{+}(T, \operatorname{cc}(E, F))$ converges in the $w n$-topology to $M$ if and only if $\left(M_{i}(T)\right)$ converges to $M(T)$ in $\operatorname{cc}(E, F)$ and for every $y \in F$ and every $f \in C_{+}(T),\left(\int f \delta^{*}\left(y \mid M_{i}(\cdot)\right)\right)$ converges to $\int f \delta^{*}(y \mid M(\cdot))$. It follows that if $T$ is a completely regular space, the $w n$-topology in $\widetilde{M}_{+}(T, \mathcal{K}(T), \operatorname{cc}(E, F))$ is a uniform topology. The uniformity is generated by the families of pseudometrics $\left\{p_{V} ; V \in \beta(o)\right\}$ and $\left\{p_{f, y} ; y \in F, f \in C_{+}(T)\right\}$, defined as follows: for every $M$ and $M^{\prime}$ in $\widetilde{M}_{+}(T, \mathcal{K}(T), \operatorname{cc}(E, F))$

$$
\begin{aligned}
p_{V}\left(M, M^{\prime}\right) & =\sup _{\substack{\circ \in V \\
\circ}}\left|\delta^{*}(y \mid M(T))-\delta^{*}\left(y \mid M^{\prime}(T)\right)\right|, \\
p_{f, y}\left(M, M^{\prime}\right) & =\left|\int f \delta^{*}(y \mid M(\cdot))-\int f \delta^{*}\left(y \mid M^{\prime}(\cdot)\right)\right| .
\end{aligned}
$$

It is evident that $\widetilde{M}_{+}(T, \mathcal{K}(T), \operatorname{cc}(E, F))$ endowed with this uniform topology is a Hausdorff space.

Let us introduce another topology. The simple topology ( $s$-topology) on $\widetilde{M}_{+}(T, \operatorname{cc}(E, F))$ is the weakest topology for which all maps $M \mapsto M(f)$ are continuous for every $f \in C_{+}(T)$.

2. Main results. The following theorem was proved by Topsøe ([16, Theorem 4, p. 202]) for non-negative scalar measures.

TheOrem 2.1. Let $\mathcal{G}$ and $\mathcal{K}$ be families of subsets of a set $T$ which satisfy axioms (i)-(v) and let $H$ be a subset of $\widetilde{M}_{+}(T, \mathcal{K}, \operatorname{cc}(E, F))$ endowed with the wn-topology. Then the following conditions (1) and (2) are equivalent:

(1) Every net on $H$ has a convergent subnet in $\widetilde{M}_{+}(T, \mathcal{K}, \operatorname{cc}(E, F))$.

(2) (a) The set $\{M(T) ; M \in H\}$ is relatively compact in $\operatorname{cc}(E, F)$.

(b) For every $y \in F$, every subclass $\mathcal{G}^{\prime}$ of $\mathcal{G}$ which dominates $\mathcal{K}$, and every $\varepsilon>0$ there exists a finite subclass $\mathcal{G}^{\prime \prime}$ of $\mathcal{G}^{\prime}$ such that

$$
\sup _{M \in H} \inf _{G \in \mathcal{G}^{\prime \prime}} \delta^{*}(y \mid M(T \backslash G))<\varepsilon .
$$

Proof. Assume that (1) is satisfied. It is obvious that (a) holds. If (b) failed we would find $y \in F, \varepsilon>0, \mathcal{G}^{\prime} \subseteq \mathcal{G}$ with $\mathcal{G}^{\prime} \succ \mathcal{K}$ such that for any finite subfamily $\mathcal{G}^{\prime \prime}$ of $\mathcal{G}^{\prime}$ there exists $M_{\mathcal{G}^{\prime \prime}} \in H$ such that $\inf \left\{\delta^{*}\left(y \mid M_{\mathcal{G}^{\prime \prime}}(T \backslash G)\right)\right.$; $\left.G \in \mathcal{G}^{\prime \prime}\right\} \geq \varepsilon$. We then obtain a net $\left(M_{\mathcal{G}^{\prime \prime}}\right)_{\mathcal{G}^{\prime \prime} \subset \mathcal{G}^{\prime}}$, where the family of all 
finite subsets of $\mathcal{G}^{\prime}$ is directed by $\supset$. According to (1), the net $M_{\mathcal{G}^{\prime \prime}}$ has a subnet convergent in $\widetilde{M}_{+}(T, \mathcal{K}, \operatorname{cc}(E, F))$. We denote this subnet again by $\left(M_{\mathcal{G}^{\prime \prime}}\right)$; let $M$ be its limit. Then

$$
\begin{aligned}
\lim _{\mathcal{G}^{\prime \prime}} \delta^{*} & \left(y \mid M_{\mathcal{G}^{\prime \prime}}(T)\right) \\
& =\delta^{*}(y \mid M(T))=\sup _{K \in \mathcal{K}} \delta^{*}(y \mid M(K)) \leq \sup _{K \in \mathcal{K}} \inf _{G \in \mathcal{G}^{\prime}, K \subset G} \delta^{*}(y \mid M(G)) \\
& \leq \sup _{K \in \mathcal{K}} \inf _{G \in \mathcal{G}^{\prime}, K \subset G} \liminf _{\mathcal{G}^{\prime \prime}} \delta^{*}\left(y \mid M_{\mathcal{G}^{\prime \prime}}(G)\right) \\
& =\sup _{K \in \mathcal{K}} \inf _{K \subseteq G, G \in \mathcal{G}^{\prime}} \liminf _{\mathcal{G}^{\prime \prime}}\left[\delta^{*}\left(y \mid M_{\mathcal{G}^{\prime \prime}}(T)\right)-\delta^{*}\left(y \mid M_{\mathcal{G}^{\prime \prime}}(T \backslash G)\right)\right] \\
& =\sup _{K \in \mathcal{K}} \inf _{K \subseteq G, G \in \mathcal{G}^{\prime}}\left[\lim _{\mathcal{G}^{\prime \prime}} \delta^{*}\left(y \mid M_{\mathcal{G}^{\prime \prime}}(T)\right)-\limsup _{\mathcal{G}^{\prime \prime}} \delta^{*}\left(y \mid M_{\mathcal{G}^{\prime \prime}}(T \backslash G)\right)\right] \\
& \leq \lim _{\mathcal{G}^{\prime \prime}} \delta^{*}\left(y \mid M_{\mathcal{G}^{\prime \prime}}(T)\right)-\varepsilon,
\end{aligned}
$$

a contradiction.

Let us now prove the converse. Assume that (2) is satisfied. It suffices to prove that every universal net $\left(M_{i}\right)_{i \in I}$ on $H$ converges in $\widetilde{M}_{+}(T, \mathcal{K}, \operatorname{cc}(E, F)$ ). Because of $(2 \mathrm{a}),\left(M_{i}(T)\right)_{i \in I}$ is convergent in $\operatorname{cc}(E, F) . \operatorname{Put}_{i} \lim _{i}(T)=$ $C$. The set $\bigcup\{M(T) ; M \in H\}$ is bounded in $E$. So is $\bigcup\{M(A) ; M \in$ $H\}$ for every $A \in \mathcal{B}$ because $M(A) \subset M(T)$. Then for each $y \in F$ and $A \in \mathcal{B}$ the universal net $\left(\delta^{*}\left(y \mid M_{i}(A)\right)\right)_{i \in I}$ is convergent in $\mathbb{R}$. Put $p_{y}(A)=$ $\lim _{i} \delta^{*}\left(y \mid M_{i}(A)\right)$. Let $G \in \mathcal{G}$. Define $S_{G}: F \rightarrow \mathbb{R}$ by $S_{G}(y)=p_{y}(G)$. One has $S_{G}\left(y+y^{\prime}\right) \leq S_{G}(y)+S_{G}\left(y^{\prime}\right)$ and $S_{G}(\alpha y)=\alpha S_{G}(y)$ for all $\alpha \geq 0$ and $y, y^{\prime} \in F$, and $\left|S_{G}(y)\right| \leq \delta^{*}(y \mid \widetilde{C})$ where $\widetilde{C}$ is the absolutely convex hull of $C$. We have $\widetilde{C} \in \operatorname{cc}(E, F)$ ([7, p. 242]). This proves that $S_{G}$ is $\sigma(F, E)$-continuous. By the Hahn-Banach theorem $([4$, p. 62]) we have $S_{G}(y)=\sup \left\{l_{G}(y) ; l_{G}: F \rightarrow \mathbb{R}\right.$ linear and $\left.l_{G} \leq S_{G}\right\}$. The relation $l_{G} \leq S_{G}$ shows that $l_{G}$ is also $\sigma(F, E)$-continuous. Hence we may put $l_{G}(y)=y\left(x_{G}\right)$ where $x_{G} \in E$. Denote by $\operatorname{cf}(E, F)$ the set of all convex closed non-empty subsets of $E$ and consider the map

$$
M: \mathcal{G} \rightarrow \operatorname{cf}(E), \quad G \mapsto M(G)=\operatorname{cl}\left\{x_{G} ; x_{G} \in E, \forall y \in F y\left(x_{G}\right) \leq S_{G}(y)\right\} .
$$

One has $S_{G}(y)=\delta^{*}(y \mid M(G))$. Since $S_{G}(y) \leq \delta^{*}(y \mid \widetilde{C})$ for every $y \in F$ we have $M(G) \in \operatorname{cc}(E, F)$. Moreover, $M$ is positive, monotone and subadditive. In view of [12, Theorem 2] the map $\widetilde{M}$ from $\mathcal{B}$ to $\operatorname{cf}(E, F)$ defined by

$$
\widetilde{M}(A)=\overline{\mathrm{co}} \bigcup_{K \subseteq A} \bigcap_{G \supseteq K} M(G)
$$

is a positive weak set-valued measure. It is $\mathcal{K}$-inner regular and $\widetilde{M}(G) \subseteq$ $M(G)$ for every $G \in \mathcal{G}$. Since $M(G) \subseteq \widetilde{C}$ for all $G \in \mathcal{G}$, we have $\widetilde{M}(A) \in$ $\operatorname{cc}(E, F)$ for all $A \in \mathcal{B}$. 
Let us prove that $\left(M_{i}\right)_{i \in I}$ converges to $\widetilde{M}$. By the definition of $M$, we have $\lim _{i} \delta^{*}\left(y \mid M_{i}(G)\right)=\delta^{*}(y \mid M(G))$. Since $\widetilde{M}(G) \subseteq M(G)$,

$$
\forall y \in F, \forall G \in \mathcal{G}, \quad \lim _{i} \delta^{*}\left(y \mid M_{i}(G)\right) \geq \delta^{*}(y \mid \widetilde{M}(G)) .
$$

It remains to show that $\lim _{i} M_{i}(T)=\widetilde{M}(T)$. First let us prove that $\lim _{i} \delta^{*}\left(y \mid M_{i}(T)\right)=\delta^{*}(y \mid \widetilde{M}(T))$ for all $y \in F$. Note that

$$
\delta^{*}(y \mid \widetilde{M}(T))=\sup _{K \in \mathcal{K}} \inf _{G \supseteq K} \delta^{*}(y \mid M(G))
$$

([12, Lemmas 1-3]). Therefore we have to prove that

$$
\forall y \in F \quad \inf _{K \in \mathcal{K}} \sup _{K \subseteq G} \lim _{i} \delta^{*}\left(y \mid M_{i}(T \backslash G)\right)=0 .
$$

If this were not so we would find $\varepsilon>0$ and $y \in F$ such that for every $K \in \mathcal{K}$ there exists $G_{K} \in \mathcal{G}$ with $G_{K} \supset K$ and $\delta^{*}\left(y \mid M_{i}\left(T \backslash G_{K}\right)\right)>\varepsilon$ eventually. Put $\mathcal{G}^{\prime}=\left\{G_{K} ; K \in \mathcal{K}\right\}$; then $\mathcal{G}^{\prime}$ dominates $\mathcal{K}$ and for every finite subfamily $\mathcal{G}^{\prime \prime}$ of $\mathcal{G}^{\prime}$ we have $\inf \left\{\delta^{*}\left(y \mid M_{i}\left(T \backslash G_{K}\right)\right) ; G_{K} \in \mathcal{G}^{\prime \prime}\right\}>\varepsilon$ eventually. This contradicts condition (b) of (2). Therefore $\lim _{i} \delta^{*}\left(y \mid M_{i}(T)\right)=\delta^{*}(y \mid \widetilde{M}(T))$ for all $y \in F$.

On the other hand, the net $\left(M_{i}(T)\right)$ converges to $C$ in $\operatorname{cc}(E, F)$. It follows that $\delta^{*}(y \mid C)=\delta^{*}(y \mid \widetilde{M}(T))$ for all $y \in F$ and therefore $\widetilde{M}(T)=C$.

REMARK. If in condition (2)(b) we only take subclasses $\mathcal{G}^{\prime \prime}$ of $\mathcal{G}^{\prime}$ consisting of one set then we obtain the following condition:

(3) For all $y \in F$, all $\mathcal{G}^{\prime} \subset \mathcal{G}$ with $\mathcal{G}^{\prime} \succ \mathcal{K}$ and all $\varepsilon>0$ there exists $G \in \mathcal{G}^{\prime}$ such that

$$
\sup \left\{\delta^{*}(y \mid M(T \backslash G)) ; M \in H\right\}<\varepsilon .
$$

In view of $[14$, Lemma 7$]$ this condition is equivalent to the following:

(4) For all $y \in F$ and $\varepsilon>0$ there exists $K \in \mathcal{K}$ such that

$$
\sup \left\{\delta^{*}(y \mid M(T \backslash K)) ; M \in H\right\}<\varepsilon .
$$

Definition. A subset of $\widetilde{M}_{+}(T, \mathcal{K}, \operatorname{cc}(E, F))$ which satisfies condition (4) is said to be uniformly tight.

COROllary 2.1. Let $T$ be an abstract set, and let $\mathcal{G}$ and $\mathcal{K}$ be families of subsets of $T$ which satisfy axioms (i)-(v). Let $H \subset \widetilde{M}_{+}(T, \mathcal{K}, \operatorname{cc}(E, F))$ be such that $\{M(T) ; M \in H\}$ is relatively compact in $\operatorname{cc}(E, F)$. If $H$ is uniformly tight, then every net on $H$ has a convergent subnet.

The results of the next corollary have been proved in [6] for scalar-valued measures. For non-negative measures they have been proved separately by several authors (e.g. [3], [1], [8], [18], [9], [5]). We have generalized them to set-valued measures [13]. 
COROllary 2.2. Assume that $T$ is a locally compact space or a complete metric space or else a hemicompact $k$-space. Let $H$ be a subset of $\widetilde{M}_{+}(T, \mathcal{K}(T), \operatorname{cc}(E, F))$ endowed with the wn-topology. Then the following conditions (1) and (2) are equivalent:

(1) $H$ is relatively compact.

(2) (a) The set $\{M(T) ; M \in H\}$ is relatively compact in $\operatorname{cc}(E, F)$,

(b) $H$ is uniformly tight.

Since $\widetilde{M}_{+}(T, \mathcal{K}(T), \operatorname{cc}(E, F))$ is a completely regular space, condition (1) of the corollary is equivalent to that of the theorem. The result is evident if $T$ is a locally compact space or a complete metric space. If $T$ is a hemicompact $k$-space the proof is similar to that in [9, Theorem 5.2, p. 884].

Finally, note that $\widetilde{M}_{+}(T, \mathcal{K}, \operatorname{cc}(E, F))$ with the $w n$-topology is a Hausdorff space when axioms (i)-(v) are satisfied. Since two weak set-valued measures $M$ and $M^{\prime}$ are equal if and only if $\delta^{*}(y \mid M(\cdot))=\delta^{*}\left(y \mid M^{\prime}(\cdot)\right)$ for all $y \in F$, the proof follows from that of Topsøe ([16, p. 204]).

3. The space $\widetilde{M}_{+}(T, \mathcal{K}(T), \operatorname{ck}(E))$. In this section we prove that the $w n$-topology, the $s n$-topology and the $s$-topology coincide in $\widetilde{M}_{+}(T, \mathcal{K}(T)$, $\operatorname{ck}(E))$. Now $E$ is a Banach space and $F=E^{\prime}$ is its topological dual. The norms on $E$ and $E^{\prime}$ are denoted by $|\cdot|$. Let $B^{\prime}(0,1)$ be the closed unit ball of $E^{\prime}$, endowed with the relative topology $\sigma\left(B^{\prime}(0,1), E\right)$ generated by the weak topology $\sigma\left(E^{\prime}, E\right)$ in $E^{\prime}$. We denote by $\operatorname{ck}(E)$ the space of all convex compact non-empty subsets of $E$, and by $\widetilde{M}_{+}(T, \mathcal{K}(T), \operatorname{ck}(E))$ the subspace of $\widetilde{M}_{+}\left(T, \mathcal{K}(T), \operatorname{cc}\left(E, E^{\prime}\right)\right)$ consisting of all elements with values in $\operatorname{ck}(E)$. Note that a weak set-valued $M$ with values in $\operatorname{cc}\left(E, E^{\prime}\right)$ is a setvalued measure, that is, for any sequence $\left(A_{n}\right)$ of pairwise disjoint sets in $\mathcal{B}(T)$ with union $A$, we have $M(A)=\lim _{n \rightarrow \infty} \sum_{k=0}^{n} M\left(A_{k}\right)$ where the limit is taken with respect to the Hausdorff topology [15]. The Hausdorff topology derives from the distance $\delta$ defined by $\delta\left(C, C^{\prime}\right)=\sup \left\{\left|\delta^{*}(y \mid C)-\delta^{*}\left(y \mid C^{\prime}\right)\right|\right.$; $\left.y \in E^{\prime},|y| \leq 1\right\}$ for all $C$ and $C^{\prime}$ in $\operatorname{cc}\left(E, E^{\prime}\right)$. The space $(\operatorname{ck}(E), \delta)$ is a complete metric space [2]. We start with the following

Lemma 3.1. Let $\left(C_{i}\right)_{i \in I}$ be a net on $\operatorname{ck}(E)$, and let $\left(z_{i}\right)_{i \in I}$ be a net on $B^{\prime}(0,1)$. If $\left(C_{i}\right)$ converges to $C_{0}$ in $\mathrm{ck}(E)$, and $\left(z_{i}\right)$ converges to $z_{0}$ in $B^{\prime}(0,1)$, then $\left(\delta^{*}\left(z_{i} \mid C_{i}\right)\right)$ converges to $\delta^{*}\left(z_{0} \mid C_{0}\right)$.

Proof. We have

$$
\begin{aligned}
\left|\delta^{*}\left(z_{i} \mid C_{i}\right)-\delta^{*}\left(z_{0} \mid C_{0}\right)\right| & \leq\left|\delta^{*}\left(z_{i} \mid C_{i}\right)-\delta^{*}\left(z_{i} \mid C_{0}\right)\right|+\left|\delta^{*}\left(z_{i} \mid C_{0}\right)-\delta^{*}\left(z_{0} \mid C_{0}\right)\right| \\
& \leq \sup _{|y| \leq 1}\left|\delta^{*}\left(y \mid C_{i}\right)-\delta^{*}\left(y \mid C_{0}\right)\right|+\left|\delta^{*}\left(z_{i} \mid C_{0}\right)-\delta^{*}\left(z_{0} \mid C_{0}\right)\right| .
\end{aligned}
$$

Since $C_{0} \in \operatorname{ck}(E)$ and the map $\delta^{*}\left(\cdot \mid C_{0}\right): B^{\prime}(0,1) \rightarrow \mathbb{R}, y \mapsto \delta^{*}\left(y \mid C_{0}\right)$, is 
continuous, the net $\left(\delta^{*}\left(z_{i} \mid C_{0}\right)\right)_{i}$ converges to $\delta^{*}\left(z_{0} \mid C_{0}\right)$. Moreover

$$
\lim _{i}\left(\sup _{|y| \leq 1}\left|\delta^{*}\left(y \mid C_{i}\right)-\delta^{*}\left(y \mid C_{0}\right)\right|\right)=0
$$

because $\left(C_{i}\right)$ converges to $C_{0}$. The lemma is therefore proved.

TheOREM 3.2. Let $T$ be a completely regular Hausdorff space, and $E$ be a Banach space. Let $\left(M_{i}\right)_{i \in I}$ be a net on $\widetilde{M}_{+}(T, \mathcal{K}(T), \operatorname{ck}(E))$ and $M_{0} \in$ $\widetilde{M}_{+}(T, \mathcal{K}(T), \operatorname{ck}(E))$. Then $\left(M_{i}\right)$ converges to $M_{0}$ in the wn-topology if and only if $\left(M_{i}\right)$ converges to $M_{0}$ in the s-topology.

Proof. By Section 1.6 it is evident that the $s$-topology is finer than the $w n$-topology, so we need only prove that convergence in the $w n$-topology implies convergence in the $s$-topology. Assume that $\left(M_{i}\right)$ converges to $M_{0}$ in the $w n$-topology. To show that $\left(M_{i}\right)$ converges to $M_{0}$ in the $s$-topology it suffices to prove that for every $f \in C_{+}(T),\left(\int f M_{i}\right)$ is a Cauchy net. If this were not so, there would exist $g \in C_{+}(T)$ and $\varepsilon>0$ such that for every $i \in I$ we would find $k_{i}, j_{i} \in I$ with $k_{i}, j_{i} \geq i$ and $y_{i} \in B^{\prime}(0,1)$ such that $\left|\int g \delta^{*}\left(y_{i} \mid M_{j_{i}}(\cdot)\right)-\int g \delta^{*}\left(y_{i} \mid M_{k_{i}}(\cdot)\right)\right| \geq \varepsilon$. We may assume without loss of generality that $g \leq 1$. Since $B^{\prime}(0,1)$ is a compact space for the topology $\sigma\left(B^{\prime}(0,1), E\right)$, the net $\left(y_{i}\right)_{i \in I}$ has a convergent subnet. Assume for simplicity that $\left(y_{i}\right)$ itself converges to $z \in B^{\prime}(0,1)$. Consider the net $\left(\int g \delta^{*}\left(y_{i} \mid M_{k_{i}}(\cdot)\right)\right)_{i \in I}$. We have

$$
\int g \delta^{*}\left(y_{i} \mid M_{k_{i}}(\cdot)\right)-\int g \delta^{*}\left(z \mid M_{k_{i}}(\cdot)\right) \leq \int g \delta^{*}\left(y_{i}-z \mid M_{k_{i}}(\cdot)\right)
$$

because for every $y$ and $y^{\prime}$ in $E^{\prime}$ one has $\int g \delta^{*}\left(y+y^{\prime} \mid M_{k_{i}}(\cdot)\right) \leq \int g \delta^{*}\left(y \mid M_{k_{i}}(\cdot)\right)$ $+\int g \delta^{*}\left(y^{\prime} \mid M_{k_{i}}(\cdot)\right)$. Since $g \leq 1$ and $\delta^{*}\left(y \mid M_{k_{i}}(\cdot)\right)$ is a non-negative measure one has $\int g \delta^{*}\left(y \mid M_{k_{i}}(\cdot)\right) \leq \delta^{*}\left(y \mid M_{k_{i}}(T)\right)$ for every $y \in E^{\prime}$. We then have

$$
\int g \delta^{*}\left(y_{i} \mid M_{k_{i}}(\cdot)\right)-\int g \delta^{*}\left(z \mid M_{k_{i}}(\cdot)\right) \leq 2 \delta^{*}\left(\frac{1}{2}\left(y_{i}-z\right) \mid M_{k_{i}}(T)\right) .
$$

It follows that

$$
\begin{aligned}
& \left|\int g \delta^{*}\left(y_{i} \mid M_{k_{i}}(\cdot)\right)-\int g \delta^{*}\left(z \mid M_{k_{i}}(\cdot)\right)\right| \\
& \quad \leq 2 \sup \left(\delta^{*}\left(\frac{1}{2}\left(y_{i}-z\right) \mid M_{k_{i}}(T)\right), \delta^{*}\left(\frac{1}{2}\left(z-y_{i}\right) \mid M_{k_{i}}(T)\right)\right) .
\end{aligned}
$$

By Lemma 3.1 the nets $\left(\delta^{*}\left(\frac{1}{2}\left(y_{i}-z\right) \mid M_{k_{i}}(T)\right)\right)_{i}$ and $\left(\delta^{*}\left(\frac{1}{2}\left(z-y_{i}\right) \mid M_{k_{i}}(T)\right)\right)_{i}$ converge to 0 . Hence $\lim _{i}\left|\int g \delta^{*}\left(y_{i} \mid M_{k_{i}}(\cdot)\right)-\int g \delta^{*}\left(z \mid M_{k_{i}}(\cdot)\right)\right|=0$. Taking account of the hypothesis one has $\lim _{i} \int g \delta^{*}\left(z \mid M_{k_{i}}(\cdot)\right)=\int g \delta^{*}\left(z \mid M_{0}(\cdot)\right)$. Then we may conclude that $\lim _{i} \int g \delta^{*}\left(y_{i} \mid M_{k_{i}}(\cdot)\right)=\int g \delta^{*}\left(z \mid M_{0}(\cdot)\right)$. Analogously, $\lim _{i} \int g \delta^{*}\left(y_{i} \mid M_{j_{i}}(\cdot)\right)=\int g \delta^{*}\left(z \mid M_{0}(\cdot)\right)$. It follows from the equality of those limits that $\lim _{i}\left|\int g \delta^{*}\left(y_{i} \mid M_{k_{i}}(\cdot)\right)-\int g \delta^{*}\left(y_{i} \mid M_{j_{i}}(\cdot)\right)\right|=0$. That is a contradiction. 
We denote by $t_{s n}, t_{w n}$ and $t_{s}$ the strong-narrow, weak-narrow and simple topology, respectively. If $t$ and $t^{\prime}$ are two topologies on the same set, we write $t \preceq t^{\prime}$ if $t$ is coarser than $t^{\prime}$.

Let $G \in \mathcal{G}(T)$ and $K \in \mathcal{K}(T)$ and assume that $K \subset G$ and $T$ is a completely regular Hausdorff space. Put $\mathcal{F}=\left\{f \in C_{+}(T) ; f<1_{G}\right\}$ where $1_{G}$ is the indicator function of $G$. Since $T$ is a completely regular Hausdorff space, there exists $f \in \mathcal{F}$ such that $f(x)=1$ for all $x \in K$. The family $\mathcal{F}$ is filtering to the right and $1_{G}=\sup \{f ; f \in \mathcal{F}\}$. Now let $M \in \widetilde{M}_{+}(T, \mathcal{K}(T), \operatorname{ck}(E))$. Since $M$ is $\mathcal{K}(T)$-inner regular and positive, we have $M(G)=\overline{\operatorname{co}} \bigcup\left\{\int f M ; f \in \mathcal{F}\right\}=\operatorname{cl} \bigcup\left\{\int f M ; f \in \mathcal{F}\right\}$. The second equality follows from the fact that $\bigcup\left\{\int f M ; f \in \mathcal{F}\right\}$ is a convex set in $E$. Indeed, if $x \in \int f M$ and $y \in \int g M$ with $f \in \mathcal{F}$ and $g \in \mathcal{F}$, then $h=\sup (f, g) \in \mathcal{F}$ and $M(h) \supseteq M(f) \cup M(g)$ because $M$ is positive. Therefore $r x+(1-r) y \in M(h)$ where $0 \leq r \leq 1$.

THEOREM 3.3. Let $T$ be a completely regular Hausdorff space and let $E$ be a Banach space. Then in $\widetilde{M}_{+}(T, \mathcal{K}(T), \operatorname{ck}(E))$ the wn-topology, sntopology and s-topology are identical.

Proof. Let $f \in C_{+}(T)$ and let

$$
p_{f}:\left(\widetilde{M}_{+}(T ; \mathcal{K}(T), \operatorname{ck}(E)), t_{s}\right) \rightarrow(\operatorname{ck}(E), \delta), \quad M \mapsto p_{f}(M)=\int f M .
$$

Then $p_{f}$ is continuous, and therefore lower semicontinuous. It follows that for every $G \in \mathcal{G}(T)$ the map

$$
\begin{gathered}
p_{G}:\left(\widetilde{M}_{+}(T, \mathcal{K}(T), \operatorname{ck}(E)), t_{s}\right) \rightarrow(\operatorname{ck}(E), \delta) \\
M \mapsto p_{G}(M)=M(G)=\operatorname{cl} \bigcup\left\{\int f M ; f \in \mathcal{F}\right\}
\end{gathered}
$$

is lower semicontinuous. We deduce that $t_{s n} \preceq t_{s}$ because $t_{s n}$ is the weakest topology for which all maps $M \mapsto M(G)$ defined on $\widetilde{M}_{+}(T, \mathcal{K}(T), \operatorname{ck}(E))$ are lower semicontinuous. It follows from the proof of Theorem II.21 in [2, p. 52] that $t_{w n} \preceq t_{s n}$. The relations $t_{w n} \preceq t_{s n} \preceq t_{s}$ and Theorem 3.2 show that these three topologies are identical.

\section{References}

[1] N. Bourbaki, Intégration, chapitre IX, Hermann, Paris.

[2] C. Castaing and M. Valadier, Convex Analysis and Measurable Multifunctions, Lecture Notes in Math. 580, Springer, 1977.

[3] J. B. Conway, The strict topology and compactness in the space of measures, Trans. Amer. Math. Soc. 126 (1967), 474-486.

[4] N. Dunford and J. T. Schwartz, Linear Operators, Part I, General Theory, Interscience, New York, 1958.

[5] X. Fernique, Processus linéaires, processus généralisés, Ann. Inst. Fourier (Grenoble) 17 (1967), no. 1, 1-92. 
[6] D. H. Fremlin, D. J. H. Garling and R. G. Haydon, Bounded measures on topological spaces, Proc. London Math. Soc. (3) 25 (1972), 115-136.

[7] G. Köthe, Topological Vector Spaces I, 2nd ed., Springer, Berlin, 1983.

[8] L. Le Cam, Convergence in distribution of stochastic processes, Univ. Calif. Publ. Statist. 2 (1957), 207-236.

[9] S. E. Mosiman and R. F. Wheeler, The strict topology in a completely regular setting: Relations to topological measure theory, Canad. J. Math. 24 (1972), 873-890.

[10] D. Preiss, Metric spaces in which Prokhorov's theorem is not valid, Z. Wahrsch. Verw. Gebiete 27 (1973), 109-116.

[11] Yu. V. Prokhorov, Convergence of random processes and limit theorems in probability theory, Theor. Probab. Appl. 1 (1956), 157-216.

[12] K. K. Siggini, On the construction of set-valued measures, Bull. Polish Acad. Sci. Math. 51 (2003), 251-259.

[13] —, Compacité étroite des multi-applications tendues, Rev. Roumaine Math. Pures Appl. 33 (1988), 457-470.

[14] —, Sur la compacité des multimesures I, C. R. Math. Acad. Sci. Paris 334 (2002), 949-952.

[15] D. S. Thiam, Intégration dans les espaces ordonnés et intégration multivoque, thèse, Univ. Pierre et Marie Curie, 1976.

[16] F. Topsøe, Compactness in spaces of measures, Studia Math. 36 (1970), 195-212.

[17] - Topology and Measure, Lecture Notes in Math. 133, Springer, Berlin, 1970.

[18] V. S. Varadarajan, Measures on topological spaces, Mat. Sb. 55 (1961), 35-100 (in Russian); English transl.: Amer. Math. Soc. Transl. Ser. II 48 (1965), 161-228.

Kenny Koffi Siggini

Department of Mathematics

Faculty of Sciences

University of Lome

P.O. Box 1515, Lome, Togo

E-mail: ksiggini@hotmail.com 